\title{
Prevalence and factors associated to dysphonia and laryngeal lesions: a study among teachers in a region of the Brazilian Legal Amazon
}

\author{
Prevalência e fatores associados à disfonia e lesões laríngeas: um estudo entre \\ professores de uma região da Amazônia Legal Brasileira
}

Fernanda Aguiar da $\mathrm{Cruz}^{1}$, Adriana Sousa Rêgo², Wellyson da Cunha Araújo Firmo³ ${ }^{2}$ Daniela Bassi-Dibai ${ }^{4}$, Flor de Maria Araujo Mendonça Silva ${ }^{5}$, Ilana Mírian Almeida Felipe da Silva ${ }^{6 *}$

\begin{abstract}
${ }^{1}$ Mestre pelo Programa de Pós-graduação em Gestão de Programa e Serviços de Saúde pela Universidade CEUMA, MA; ${ }^{2}$ Doutora pelo Programa de Pós-graduação em Saúde Coletiva pela Universidade Federal do Maranhão (UFMA), Docente do Programa de Pós-graduação em Meio Ambiente (UNIVERSIDADE CEUMA); ${ }^{3}$ Doutor pelo Programa de Pós-graduação em Biodiversidade e Biotecnologia pela UFMA, Docente do Programa de Pós-graduação em Biologia Microbiana a UNIVERSIDADE CEUMA; ${ }^{4}$ Doutora pelo Programa de Pós-graduação em Fisioterapia pela Universidade Federal de São Carlos (UFSCar). Docente do Programa de Pós-graduação em Gestão de Programa e Serviços de Saúde pela UNIVERSIDADE CEUMA; ${ }^{5}$ Doutora pelo Programa de Pós-graduação em Saúde Coletiva pela UFMA, Docente do Programa de Pós-graduação em Gestão de Programa e Serviços de Saúde da UNIVERSIDADE CEUMA; ${ }^{6}$ Doutora pelo Programa de Pós-graduação em Enfermagem em Saúde Pública pela Universidade de São Paulo USP), Docente do

Programa de Pós-graduação em Gestão de Programa e Serviços de Saúde da UNIVERSIDADE CEUMA
\end{abstract}

\begin{abstract}
Objective: to investigate the prevalence and factors associated with dysphonia and laryngeal lesions at elementary public school teachers in a region of the Amazonian region. Methodology: cross-sectional study with collection of sociodemographic, occupational, environmental, and clinics variables. Dysphonia and laryngeal lesions were evaluated using three methods: self-perceptions, diagnostic criteria and video-assisted laryngoscopy. Logistic regression was used to determine factors associated. Results: the prevalence of self-reported dysphonia was $68.9 \%$, diagnosed dysphonia was $59.1 \%$ and laryngeal lesions, $53.3 \%$. There was association between selfreported dysphonia and respiratory problems (OR=3.00; $C 195 \%=1.25-7.22)$, use of drugs $(O R=2.31, C 195 \%=1.19-4.49)$ and noise in the internal environment (OR=2.43; $C 195 \%=1.24-4.73)$. Easy access to water was a protection factor against self-reported dysphonia (OR=0.09; CI95\%: 0.01-0.79). We found a significant association between dysphonia diagnosed and digestive problems (OR=5.35; $C 195 \%=2.39-11.96)$, external noise (OR=4.89, $C 195 \%=1.87-12.78$ ) and inadequate ventilation (OR=3.56; $C 195 \%=1.46-8.62)$. The variables associated with higher chance of laryngeal lesions in dysphonic teachers were lighting (OR=3.74; C195\%=1.15-12.14\%), acoustics (OR= 4.14; $C 195 \%=1.32-12.95)$ and inadequate room size $(O R=3.76 ; C 195 \%=1.29-10.91)$. The use of drugs $(O R=0.34 ; C I 95 \%=0.12-0.92)$, respiratory problems $(O R=0.21 ; C 195 \%=0.06-0.76)$ and easy access to water $(O R=0.12 ; C 195 \%=0.04-0.38)$ were protection factors for laryngeal lesions. Conclusion: occupational environmental factors aggravated the vocal health of this professional category. Access to water in the work impacted positively.

Keywords: Faculty. Dysphonia. Occupational Health. Risk Factors.
\end{abstract}

\begin{abstract}
Resumo
Objetivo: investigar a prevalência e fatores associados à disfonia e lesões laríngeas em professores do ensino fundamental de um município da Amazônia Legal. Metodologia: estudo transversal com coleta de variáveis sociodemográficas, ocupacionais, ambientais e clínicas. Disfonia e lesões laríngeas foram avaliadas com a utilização de três métodos: autopercepção, critérios diagnósticos e laringoscopia assistida por vídeo. Utilizou-se modelo de regressão logística para determinação dos fatores associados. Resultados: a prevalência da disfonia autorreferida foi $68,9 \%$, da disfonia diagnosticada foi $59,1 \%$ e das lesões laríngeas, $53,3 \%$. Houve associação entre disfonia autorreferida e problemas respiratórios $(O R=3,00$; IC95\%=1,25-7,22), uso de medicamentos (OR=2,31; IC95\%=1,19$4,49)$ e ruído interno no ambiente $(O R=2,43 ; I C 95 \%=1,24-4,73)$. Teve associação protetora entre disfonia autorreferida e facilidade do

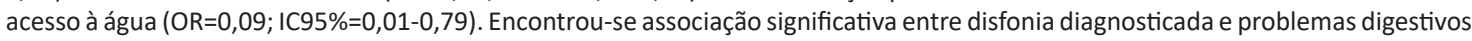
$(O R=5,35$; IC95\%=2,39-11,96), ruído externo $(O R=4,89$; IC95\%=1,87-12,78) e ventilação inadequada $(O R=3,56$; IC95\%=1,46-8,62). As variáveis associadas à maior chance de lesões laríngeas em docentes disfônicos foram iluminação $(\mathrm{OR}=3,74 ;$ IC95\%=1,15-12,14), acústica $(O R=4,14$; IC95\%=1,32-12,95) e tamanho da sala inadequados ( $O R=3,76$; IC95\%=1,29-10,91). Uso de medicação (OR=0,34; IC95\%=0,12-0,92), problemas respiratórios $(O R=0,21 ; I C 95 \%=0,06-0,76)$ e facilidade de acesso à água $(O R=0,12 ; I C 95 \%=0,04-0,38)$ mostraram-se fatores de proteção para lesões laríngeas. Conclusão: fatores ambientais ocupacionais agravaram a saúde vocal desta categoria profissional. $\mathrm{O}$ acesso à água no ambiente de trabalho impactou positivamente.
\end{abstract}

Palavras-chave: Docentes. Disfonia. Saúde do Trabalhador. Fatores de Risco.

Correspondente/Corresponding: *Ilana Mírian Almeida Felipe da Silva - End: Rua Josué Montello, No. 1, Bairro - Renascença II, São Luís - MA, 65075-120. Tel: (98) 4020-7525 - E-mail: ilana.mirian@ceuma.br 


\section{INTRODUCTION}

The teaching profession is of utmost importance to society and the teaching-learning process involves creativity and innovation ${ }^{1}$. However, teaching is frequently related to musculoskeletal, vocal, and mental disorders ${ }^{2}$. Compared to the general population, teachers are at greater risk for the development of dysphonia, which is a voice disorder due to a functional and/or organic alteration of the larynx and/or vocal tract ${ }^{3}$ associated with hoarseness, vocal fatigue, and a burning sensation in the throat'. These signs and symptoms lead to a reduction in quality of life associated with psychological problems and emotional restrictions, poor professional performance, absenteeism, reductions in social activities as well as financial costs to the health and education systems ${ }^{5}$.

Different prevalence rates of vocal disorders in this occupation are found in national and international studies, depending on the concepts and methods adopted in different investigations, such as self-perceptions, vocal signs and symptoms and video-assisted laryngoscopy ${ }^{6}$, with rates ranging from 4.4 to $90 \%{ }^{7-9}$. An American epidemiological study found that teachers have a greater prevalence rate of vocal disorders throughout their professional lives (58\%) in comparison to the general population ${ }^{10}$. A study evaluating the vocal cords of French teachers using laryngoscopy found that $20.8 \%$ had laryngeal lesions, the most frequent of which were vocal nodules ${ }^{11}$. In South Korean, the prevalence of voice disorders was significantly higher among individuals with vocal nodules $(8.1 \%$ and $27.7 \%$, respectively) 6 .

In Brazil, a study among in the private elementary and secondary school teachers, identified the prevalence of current voice problems and voice problems at some point in lifetime, with variation between $11.6 \%$ and $63 \%{ }^{12}$. In the city of São Paulo, an investigation involving educators in the municipal public system found that $60 \%$ reported vocal changes related to environmental and organizational aspects of the work, with frequent reports of mental and respiratory disorders ${ }^{13}$. In another study conducted with elementary public schools' teachers in a city in the state of São Paulo, the most prevalent vocal problems were dry throat (30.4\%), hoarseness (52.0\%), vocal fatigue (53.9\%) and shortness of breath while speaking $(81.4 \%)^{14}$. Vocal changes can occur as a result of the interaction between hereditary, individual, behavioral, lifestyle and occupational factors. The female sex, voice use pattern, high workload, environment with excessive noise and large number of students ${ }^{15,16}$, inadequate school infrastructure, absence of prior voice and communication training and how to cope with stressful situations ${ }^{17}$.

Data from the [Pesquisa Nacional por Amostra de Domicilios - PNAD], prepared by the [Instituto Brasileiro de Geografia e Estatísitca - IBGE], show that between 2002 and 2013, the number the number Brazilian schoolteachers' in the country, increased by $32.9 \%{ }^{18}$. According to [Instituto Nacional de Estudos e Pesquisas - INEP], in basic education alone there are 2.2 million teachers. considering the number of these professionals and the wage floor, it is estimated that public spending on leave, licenses and readaptations of teaching staff are $R \$ 1.66$ billion/year ${ }^{19,20}$. This is a serious public health problem, and the Brazilian Ministry of Health recognizes it as work-related voice disorder (WRVD), through the official publication of the WRVD Protoco ${ }^{19}$, which it establishes in the International Classification of Diseases, the following code (ICD-10 J04.2, J37.1, J-38, J38.2, R-49) ${ }^{21}$.

Therefore, it is important to investigate the prevalence of vocal disorders among educators with a broader vision that considers the relationship between health and work and is based on environmental and organizational conditions $^{22}$. Despite the importance of this issue, there are few studies in developing countries about vocal evaluations involving video-assisted laryngoscopy to obtain a better diagnosis $5,10,23,24$. In this perspective, the aim of the present study was to estimate the prevalence of dysphonia and laryngeal lesions and identify factors associated with these vocal problems in a municipality located in Brazilian Legal Amazon.

\section{METHODOLOGY}

An observational cross-sectional study was conducted between November 2017 and April 2018 at elementary schools in the public education system of the county of Imperatriz, state of Maranhão, which is located in the eastern region of Brazilian Amazonia. The state of Maranhão is located in the northeastern region of the country and occupies an area of $329,642.182 \mathrm{Km}^{225}$. It is necessary to emphasize that, although the county of Imperatriz is to the west of the 440 meridian, in the Legal Amazon, the state of Maranhão is located in the northeast of Brazil. The data were furnished by the Municipal Secretary of Education, which reported a total of 2619 teachers working at 159 schools in the municipality, 40 of which were exclusively nurseries, daycare centers and preschools. The remaining 119 establishments were related to elementary schools with 2221 teachers, which was the universe used to calculate the sample size. These 119 elementary schools were subdivided among five districts in the urban zone and two in the rural zone. The sample size was calculated using the following formula:

$$
n=\frac{N \cdot Z^{2} \cdot p \cdot(1-p)}{Z^{2} \cdot p \cdot(1-p)+e^{2} \cdot(N-1)}
$$

in which:

$n$-calculated sample size

$N$ - population (2221);

$Z$-determined by confidence interval $[\mathrm{Cl}=[95 \%(1.96)]$;

$p$-estimated prevalence of dysphonia: $50.0 \%$;

e-sampling error of $7 \%$.

As the literature does not offer a clear prevalence rate for dysphonia $50 \%$ was used in the calculation of the sample size. The application of the formula described 
above determined a minimum sample of 187 individuals, which was increased to 203 to compensate for a possible $10 \%$ dropout rate. Therefore, 41 teachers were selected from each of the five urban districts of county (Figure 1).

Figure 1 - Flowchart of the study steps

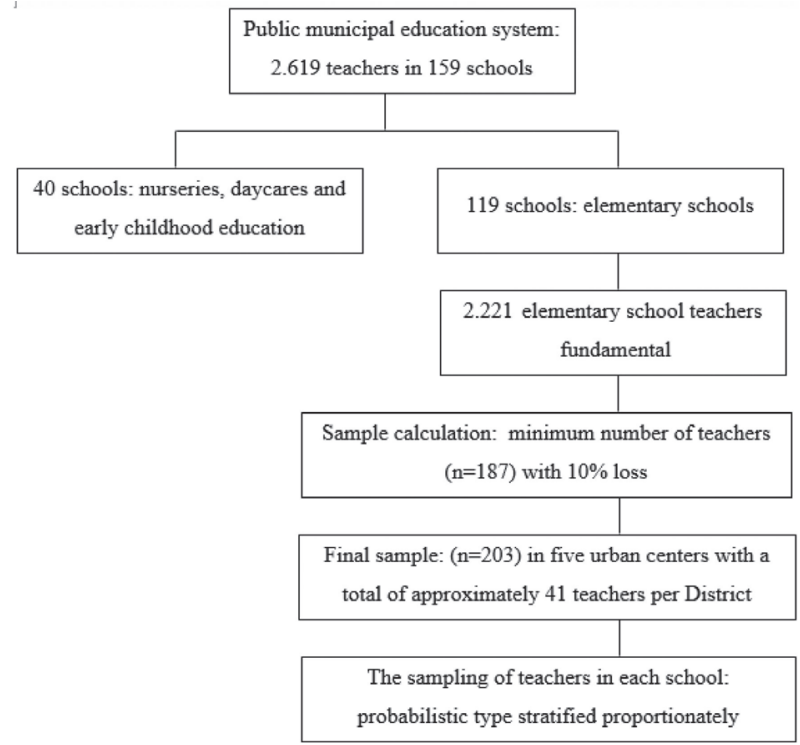

Source: Authorship

Stratified probabilistic sampling proportional to the total number of teachers per school was performed. The teachers were randomly selected from a total of four schools per district to ensure greater representativeness of the sample, this means that the total number of schools was 20 (Table 1). This process determined the number of questionnaires to be sent to each school. The questionnaires were duly coded with numbers corresponding to the probabilistic calculation and delivered to the teachers during intervals between classes. The teachers had the option of taking the questionnaires to answer at home and return on a different occasion.

Table 1 - Probabilistic sampling stratified proportionally with presentation of the four schools drawn in each District, the total number of teachers in each school and the number of questionnaires to be applied per school

\begin{tabular}{lclcc}
\hline District & $\begin{array}{c}\text { Number of } \\
\text { schools }\end{array}$ & School & $\begin{array}{c}\text { Total } \\
\text { teachers } \\
\text { (n/\%) }\end{array}$ & $\begin{array}{c}\text { Number } \\
\text { of samples } \\
\text { (n) }\end{array}$ \\
\hline Bacuri & 1 & Bacuri 1 & $22(24.2)$ & 10 \\
& 2 & Bacuri 2 & $24(26.3)$ & 11 \\
& 3 & Bacuri 3 & $25(27.5)$ & 11 \\
New Village & 4 & Bacuri 4 & $20(22.0)$ & 9 \\
& 5 & New Village 1 & $23(24.7)$ & 10 \\
& 6 & New Village 2 & $26(28.0)$ & 11 \\
& 7 & New Village 3 & $23(24.7)$ & 10 \\
& 8 & New Village 4 & $21(22.6)$ & 9
\end{tabular}

Rev. Ciênc. Méd. Biol., Salvador, v. 20, n. 1, p. 61-74, jan./abr. 2021

\begin{tabular}{lrllr} 
Lobão Village & 9 & Lobão Village 1 & $41(32.5)$ & 13 \\
& 10 & Lobão Village 2 & $40(31.7)$ & 13 \\
Santa Rita & 11 & Lobão Village 3 & $21(16.7)$ & 7 \\
& 12 & Lobão Village 4 & $24(19.1)$ & 8 \\
& 13 & Santa Rita 1 & $36(31.0)$ & 12 \\
\multirow{4}{*}{ Center } & 14 & Santa Rita 2 & $23(19.8)$ & 8 \\
& 15 & Santa Rita 3 & $34(29.3)$ & 12 \\
& 16 & Santa Rita 4 & $23(19.8)$ & 8 \\
& 17 & Center 1 & $24(22.4)$ & 9 \\
& 18 & Center 2 & $25(23.4)$ & 10 \\
& 19 & Center 3 & $30(28.0)$ & 11 \\
\hline
\end{tabular}

Source: Research data

Male and female teachers in the active phase of working at the time of the study and pertaining to the municipal public elementary school system were included in the study. Substitute teachers, assistant teachers, interns, those who had less than one year of experience teaching, those who did not make up part of the municipal public school system and those who worked in other sectors of the school and did not practice classroom teaching daily were excluded from the study. Schools in the rural zone, daycare centers and nurseries were also excluded.

Prior to the data collection process, meetings and educational lectures were held to raise the awareness of the teachers and administrators at each school. Two hundred five questionnaires were delivered and 203 were returned (99\% response rate). The study was conducted in two steps. The first step consisted of the use of an adapted self-administered questionnaire ${ }^{26}$. The following data were collected: identification (code of interviewee, school/institution, date and initials of respondent); sociodemographic characteristics (sex, age, date of birth, marital status and schooling); professional activity (teaching experience, number of schools at which the respondent was working at the time of the interview, weekly workload with students, number of students per class, grade taught, whether the respondent exercised another professional activity outside the school, type of employment tie, type of working environment [calm or not], whether interval between classes was sufficient to rest, whether the workplace offered a place for teachers to rest and number of classes the respondent taught); work environment (physical space, lighting, acoustics, ventilation, chalk, dust, external noise, internal noise [by students in the classroom] and relationship with co-workers); and general health conditions: (1) individual vocal characteristics (raising voice, shouting or using singsong voice while teaching, voice loss or failure, fatigue while speaking or at the end of the day, hoarseness, throat clearing and feeling of a lump or foreign body in throat/ oropharynx); (2) lifestyle characteristics (smoking, alcohol use, use of medications in previous two weeks, daily water 
intake and ease of access to water at school); (3) symptoms related to the respiratory system (rhinitis, sinusitis, tonsillitis, pharyngitis, laryngitis, bronchitis, asthma and colds); (4) gastric symptoms (heartburn, acid reflux and gastritis); (5) problems related to hearing system (difficulty hearing, earache, tinnitus, dizziness and/or vertigo); and (6) sleep quality.

Dysphonia and laryngeal lesions were evaluated using three methods: self-perceptions, diagnostic criteria, and video-assisted laryngoscopy. Self-reported dysphonia was assessed by reference to some general health characteristics (vocal aspects, vocal complaints, self-reported voice disorder ${ }^{27}$. The diagnostic criteria were assessed at the time of the interview with the identification of vocal characteristics compatible with 04 or more laryngopathies (cough, throat clearing, sore throat, foreign body sensation, excess mucus in the larynx, other symptoms related to the respiratory system, gastric and auditory signs, and symptoms) ${ }^{12}$.

In the second step, all teachers with self-reported dysphonia and those with vocal characteristics compatible with laryngopathy (4 or + ) were invited to participate in a specialized medical examination involving rigid video-assisted laryngoscopy performed by a head and neck surgeon and otolaryngologist. The laryngoscopic exams were scheduled through the schools using the codes of the interviewees. However, only 60 of the teachers with dysphonia underwent the exams. Laringoscopy is an exam of the upper air ways performed with a flexible fiberoptic endoscope, which enables observing the interior of the nasal and laryngeal organs. Video-assisted laryngoscopy was performed at a private medical office during previously scheduled appointments at no cost to the 60 participants who underwent this procedure. For such, a rigid $70 \circ$ optic scope with a magnification field was used, with a video camera coupled to an image capturing system of the MDmed program.

Spreadsheets on Microsoft Office Excel 2010 were used for the creation of the database. All data were entered twice for the analysis of external consistency. The items on the questionnaires were categorized in a code book. The data were expressed as absolute and relative frequencies and presented in tables. Analysis of variance was performed using the chi-squared text $\left(\chi^{2}\right)$ and Fisher's exact test for the comparison of categorical variables and the Student's t-test for the comparison of continuous variables with normal distribution.

Univariate logistic analysis was used for the selection of the main factors associated with the outcome. All variables with a $p$-value $<0.20$ in the univariate analysis were incorporated into the multivariate logistic regression analysis for the estimation of odds ratios (OR) and respective 95\% confidence intervals ( $\mathrm{Cl})$. The level of significance was set to $5 \%(p \leq 0.05)$. Simple and multiple regression models were used to quantify the association between exposure (explanatory variable) and outcome (response variable), given that the responses were binary (having or not having dysphonia; having or not having laryngeal lesions). The Statistical Package for the Social Sciences (SPSS, version 21.0, IBM Corp., Armonk, NY, USA) was used for all statistical analyses.

This study received approval from the Human Research Ethics Committee of Ceuma University (certificate number: 2.378.153/2017). All volunteers who agreed to participate in the study signed a statement of informed consent.

\section{RESULTS}

The sample was composed of 203 teachers from elementary school public system of Imperatriz, state of Maranhão, Brazil. One hundred forty exhibited dysphonia, which corresponds to a prevalence rate of $68.9 \%$, all of whom were invited to undergo video-assisted laryngoscopy, but only 60 appeared for the exams (42.8\%).

Table 2 displays the sociodemographic, professional, and environmental characteristics of the sample. One hundred twenty-five (61.6\%) considered the internal noise excessive and 84 (41.6\%) reported that the external noise was harmful; these results were statistically significant ( $p$ $=0.002$ and $p=0.027$, respectively).

Table 2 - Distribution of sociodemographic, professional, and work environment characteristics according to presence/absence of dysphonia among teachers from elementary school public system of Imperatriz, MA, Brazil, 2017 ( $n=203)$.

\begin{tabular}{|c|c|c|c|c|c|c|c|}
\hline \multirow[b]{3}{*}{ Variables } & \multicolumn{7}{|c|}{ DYSPHONIA } \\
\hline & \multicolumn{2}{|c|}{ Yes } & \multicolumn{2}{|c|}{ No } & \multicolumn{2}{|c|}{ Total } & \multirow{2}{*}{$p$-value } \\
\hline & $n \%$ & $\%$ & n \% & $\%$ & n \% & $\%$ & \\
\hline \multicolumn{8}{|l|}{ Age group ( $n=193)^{* *}$} \\
\hline$<30$ & 7 & 5.1 & 2 & 3.6 & 9 & 5.3 & \multirow{5}{*}{0.088} \\
\hline $30-39$ & 25 & 18.2 & 12 & 21.4 & 37 & 21.8 & \\
\hline $40-49$ & 54 & 39.4 & 11 & 19.6 & 65 & 38.2 & \\
\hline $50-59$ & 43 & 31.4 & 26 & 46.4 & 69 & 40.6 & \\
\hline 60 or more & 8 & 5.8 & 5 & 8.9 & 13 & 7.6 & \\
\hline $\operatorname{Sex}(n=202)^{* *}$ & & & & & & & \\
\hline
\end{tabular}


Prevalence and factors associated to dysphonia and laryngeal lesions a study among teachers in a region of the Brazilian Legal Amazon

\begin{tabular}{|c|c|c|c|c|c|c|c|}
\hline \multirow[b]{3}{*}{ Variables } & \multicolumn{7}{|c|}{ DYSPHONIA } \\
\hline & \multicolumn{2}{|c|}{ Yes } & \multicolumn{2}{|c|}{ No } & \multicolumn{2}{|c|}{ Total } & \multirow{2}{*}{$p$-value } \\
\hline & $\mathrm{n} \%$ & $\%$ & $n \%$ & $\%$ & $\mathrm{n} \%$ & $\%$ & \\
\hline Male & 19 & 13.7 & 13 & 20.6 & 32 & 15.8 & 0.209 \\
\hline Female & 120 & 86.3 & 50 & 79.4 & 170 & 84.2 & \\
\hline \multicolumn{8}{|l|}{ Marital status } \\
\hline Unknown & 4 & 2.9 & 2 & 3.2 & 6 & 3.0 & \\
\hline Married & 73 & 52.1 & 29 & 46.0 & 102 & 50.2 & 0.419 \\
\hline Divorced & 13 & 9.3 & 6 & 9.5 & 19 & 9.4 & \\
\hline Single & 48 & 34.3 & 22 & 34.9 & 70 & 34.5 & \\
\hline Widowed & 2 & 1.4 & 4 & 6.3 & 6 & 3.0 & \\
\hline \multicolumn{8}{|l|}{ Schooling } \\
\hline Teaching & 1 & 0.7 & 0 & 0.0 & 1 & 0.5 & \\
\hline High school & 2 & 1.4 & 1 & 1.6 & 3 & 1.5 & \\
\hline Master's degree & 2 & 1.4 & 0 & 0.0 & 2 & 1.0 & 0.861 \\
\hline Specialist & 46 & 32.8 & 22 & 34.9 & 68 & 32.9 & \\
\hline Higher level & 87 & 62.1 & 39 & 61.9 & 126 & 62.1 & \\
\hline Unknown & 2 & 1.4 & 1 & 1.6 & 3 & 1.5 & \\
\hline \multicolumn{8}{|c|}{ Teaching experience (years) $(n=200)^{* *}$} \\
\hline $1-5$ & 14 & 10.2 & 9 & 14.3 & 23 & 11.5 & \\
\hline $6-10$ & 24 & 17.5 & 4 & 6.3 & 28 & 14.0 & \\
\hline $11-15$ & 8 & 5.8 & 2 & 3.2 & 10 & 5.0 & \\
\hline $16-20$ & 33 & 24.1 & 19 & 30.2 & 52 & 26.0 & 0.322 \\
\hline $21-25$ & 30 & 21.9 & 18 & 28.6 & 48 & 24.0 & \\
\hline $26-30$ & 18 & 13.1 & 6 & 9.5 & 24 & 12.0 & \\
\hline$>30$ & 10 & 7.3 & 5 & 7.9 & 15 & 7.5 & \\
\hline \multicolumn{8}{|l|}{ № of schools $(n=200)^{* *}$} \\
\hline 1 & 72 & 52.6 & 30 & 47.6 & 102 & 51.0 & \\
\hline 2 & 61 & 44.5 & 32 & 50.8 & 93 & 46.5 & 0.828 \\
\hline 3 & 2 & 1.5 & 1 & 1.6 & 3 & 1.5 & \\
\hline 4 & 1 & 0.7 & 0 & 0.0 & 1 & 0.5 & \\
\hline 10 & 1 & 0.7 & 0 & 0.0 & 1 & 0.5 & \\
\hline \multicolumn{8}{|l|}{ Other activities } \\
\hline Yes & 32 & 22.9 & 7 & 11.1 & 39 & 19.2 & 0.049 \\
\hline No & 108 & 77.1 & 56 & 88.9 & 164 & 80.8 & \\
\hline \multicolumn{8}{|l|}{ Work tie } \\
\hline Contracted & 11 & 7.9 & 5 & 7.9 & 16 & 7.9 & 0.143 \\
\hline Effective & 122 & 87.1 & 57 & 90.5 & 179 & 88.2 & \\
\hline Coordinator & 7 & 5.0 & 0 & 0.0 & 7 & 3.4 & \\
\hline Other & 0 & 0.0 & 1 & 1.6 & 1 & 0.5 & \\
\hline \multicolumn{8}{|l|}{ Weekly workload (hours) } \\
\hline$<10$ & 1 & 0.7 & 2 & 3.2 & 3 & 1.5 & \\
\hline $10-20$ & 50 & 35.7 & 24 & 38.1 & 74 & 36.5 & \\
\hline $20-30$ & 6 & 4.3 & 5 & 7.9 & 11 & 5.4 & 0.498 \\
\hline $30-40$ & 73 & 52.1 & 28 & 44.4 & 101 & 49.8 & \\
\hline$>40$ & 8 & 5.7 & 4 & 6.3 & 12 & 5.9 & \\
\hline Unknown & 2 & 1.4 & 0 & 0.0 & 2 & 1.0 & \\
\hline \multicolumn{8}{|l|}{ Tranquility work environment } \\
\hline Never & 16 & 11.4 & 6 & 9.5 & 22 & 10.8 & \\
\hline
\end{tabular}


Fernanda Aguiar da Cruz et al.

\begin{tabular}{|c|c|c|c|c|c|c|c|}
\hline \multirow[b]{3}{*}{ Variables } & \multicolumn{7}{|c|}{ DYSPHONIA } \\
\hline & \multicolumn{2}{|c|}{ Yes } & \multicolumn{2}{|c|}{ No } & \multicolumn{2}{|c|}{ Total } & \multirow{2}{*}{ p-value } \\
\hline & n \% & $\%$ & $\mathrm{n} \%$ & $\%$ & $\mathrm{n} \%$ & $\%$ & \\
\hline Rarely & 45 & 32.1 & 16 & 25.4 & 61 & 30.0 & 0.765 \\
\hline Sometimes & 67 & 47.9 & 34 & 54.0 & 101 & 49.8 & \\
\hline Always & 10 & 7.1 & 5 & 7.9 & 15 & 7.4 & \\
\hline I don't know & 2 & 1.4 & 2 & 3.2 & 4 & 2.0 & \\
\hline \multicolumn{8}{|c|}{ Classroom size (number of students) } \\
\hline Unknown & 1 & 0.7 & 0 & 0.0 & 1 & 0.5 & \\
\hline$<15$ & 2 & 1.4 & 0 & 0.0 & 2 & 1.0 & 0.569 \\
\hline $15-30$ & 37 & 26.4 & 19 & 30.2 & 56 & 27.6 & \\
\hline$>30$ & 100 & 71.4 & 44 & 69.8 & 144 & 70.9 & \\
\hline \multicolumn{8}{|l|}{ Adequate place for rest } \\
\hline No & 70 & 50.0 & 38 & 60.3 & 108 & 53.2 & 0.173 \\
\hline Yes & 70 & 50.0 & 25 & 39.7 & 95 & 46.8 & \\
\hline \multicolumn{8}{|l|}{ Adequate break } \\
\hline No & 90 & 64.3 & 37 & 58.7 & 127 & 62.6 & 0.449 \\
\hline Yes & 50 & 35.7 & 26 & 41.3 & 76 & 37.4 & \\
\hline \multicolumn{8}{|l|}{ Number of classes } \\
\hline $1-5$ & 71 & 50.7 & 34 & 54.0 & 105 & 51.7 & \\
\hline $6-10$ & 40 & 28.6 & 22 & 34.9 & 62 & 30.5 & 0.252 \\
\hline$>10$ & 13 & 9.3 & 5 & 7.9 & 18 & 8.9 & \\
\hline Unknown & 16 & 11.4 & 2 & 3.2 & 18 & 8.9 & \\
\hline \multicolumn{8}{|l|}{ Ventilation } \\
\hline Adequate & 62 & 44.3 & 23 & 36.5 & 85 & 41.9 & 0.299 \\
\hline Inadequate & 78 & 55.7 & 40 & 63.5 & 118 & 58.1 & \\
\hline \multicolumn{8}{|l|}{ Lighting } \\
\hline Adequate & 86 & 61.4 & 40 & 63.5 & 126 & 62.1 & 0.779 \\
\hline Inadequate & 54 & 38.6 & 23 & 36.5 & 77 & 37.9 & \\
\hline \multicolumn{8}{|l|}{ Acoustics } \\
\hline Adequate & 64 & 45.7 & 32 & 50.8 & 96 & 47.3 & 0.655 \\
\hline Inadequate & 76 & 54.3 & 31 & 49.2 & 107 & 52.7 & \\
\hline \multicolumn{8}{|l|}{ Classroom size $(n=201)^{* *}$} \\
\hline Adequate & 104 & 75.4 & 45 & 71.4 & 149 & 74.1 & 0.556 \\
\hline Inadequate & 34 & 24.6 & 18 & 28.6 & 52 & 25.9 & \\
\hline \multicolumn{8}{|l|}{ Dust $(n=202)^{* *}$} \\
\hline No & 53 & 38.1 & 33 & 52.4 & 86 & 42.6 & 0.058 \\
\hline Yes & 86 & 61.9 & 30 & 47.6 & 116 & 57.4 & \\
\hline \multicolumn{8}{|l|}{ External noise $(n=202)^{* *}$} \\
\hline No & 74 & 53.2 & 44 & 69.8 & 118 & 58.4 & 0.027 \\
\hline Yes & 65 & 46.8 & 19 & 30.2 & 84 & 41.6 & \\
\hline \multicolumn{8}{|l|}{ Internal noise } \\
\hline No & 44 & 31.4 & 34 & 54.0 & 78 & 38.4 & 0.002 \\
\hline Yes & 96 & 68.6 & 29 & 46.0 & 125 & 61.6 & \\
\hline \multicolumn{8}{|c|}{ Good relationship with co-workers } \\
\hline No & 1 & 0.7 & 0 & 0.0 & 1 & 0.5 & 0.501 \\
\hline Yes & 139 & 99.3 & 63 & 100.0 & 202 & 99.5 & \\
\hline
\end{tabular}

Chalk dust 


\begin{tabular}{|c|c|c|c|c|c|c|c|}
\hline \multirow[b]{3}{*}{ Variables } & \multicolumn{7}{|c|}{ DYSPHONIA } \\
\hline & \multicolumn{2}{|c|}{ Yes } & \multicolumn{2}{|c|}{ No } & \multicolumn{2}{|c|}{ Total } & \multirow{2}{*}{$p$-value } \\
\hline & n \% & $\%$ & n \% & $\%$ & $\mathrm{n} \%$ & $\%$ & \\
\hline No & 139 & 99.3 & 60 & 95.2 & 199 & 98.0 & 0.055 \\
\hline Yes & 1 & 0.7 & 3 & 4.8 & 4 & 2.0 & \\
\hline
\end{tabular}

*multiple answers; **n differs from 203 due responses that were crossed out, leading to the exclusion of questionnaires only for the topic in question; Chi-squared $\left(x^{2}\right)$ test with $p \leq 0.05$, with presence/absence of dysphonia.

Source: Research data

Table 3 displays the general and vocal health characteristics of the sample. The prevalence of self-reported dysphonia was $68.9 \%(n=140)$. The prevalence of diagnosed dysphonia was $59.1 \%(n=120)$. One hundred fifty-one (74.4\%) teachers reported some type of digestive problem, $175(86.2 \%)$ reported respiratory problems and 95 (46.8\%) reported using medications; these latter two results were statistically significant $(p=0.005$ and $p=$
0.023 , respectively). Water consumption was a routine practice among 194 (95.6\%) teachers and 185 (91.1\%) reported having easy access to water at the workplace; this result was statistically significant $(p=0.003)$. The statistically significant vocal characteristics were voice loss or failure $(p<0.001)$, fatigue when speaking $(p<0.001)$ and hoarseness $(p<0.001)$.

Table 3 - Distribution of general and vocal health characteristics among teachers from elementary school public system of Imperatriz, MA, Brazil, $2017(n=203)$.

\begin{tabular}{|c|c|c|c|c|c|c|c|}
\hline \multirow[t]{3}{*}{ General health aspects } & \multicolumn{4}{|c|}{ DSYPHONIA } & \multirow{3}{*}{ Total } & \multirow{3}{*}{$\%$} & \multirow{3}{*}{$p$-value } \\
\hline & \multicolumn{2}{|c|}{ Yes } & \multicolumn{2}{|c|}{ No } & & & \\
\hline & $n \%$ & $\%$ & $n \%$ & $\%$ & & & \\
\hline Dysphonia (Self-reported) & 140 & 68.9 & 63 & 31.1 & 203 & 100 & - \\
\hline Dysphonia (Diagnosed) & 120 & 59.1 & 83 & 40.9 & 203 & 100 & - \\
\hline \multicolumn{8}{|l|}{ Digestive problems } \\
\hline Negative & 32 & 22.9 & 20 & 31.7 & 52 & 25.6 & \multirow{2}{*}{0.180} \\
\hline Positive & 108 & 77.1 & 43 & 68.3 & 151 & 74.4 & \\
\hline \multicolumn{8}{|l|}{ Respiratory problems $(n=200)^{* *}$} \\
\hline Negative & 13 & 9.3 & 15 & 23.8 & 28 & 13.8 & \multirow{2}{*}{0.005} \\
\hline Positive & 127 & 90.7 & 48 & 76.2 & 175 & 86.2 & \\
\hline \multicolumn{8}{|l|}{ Hearing problems } \\
\hline Negative & 35 & 25.0 & 15 & 23.8 & 50 & 24.6 & \multirow{2}{*}{0.855} \\
\hline Positive & 105 & 75.0 & 48 & 76.2 & 153 & 75.4 & \\
\hline \multicolumn{8}{|l|}{ Use of medication } \\
\hline No & 67 & 47.9 & 41 & 65.1 & 108 & 53.2 & \multirow{2}{*}{0.023} \\
\hline Yes & 73 & 52.1 & 22 & 34.9 & 95 & 46.8 & \\
\hline \multicolumn{8}{|l|}{ Smoking } \\
\hline No & 134 & 95.7 & 61 & 96.8 & 195 & 96.1 & \multirow{2}{*}{0.707} \\
\hline Yes & 6 & 4.3 & 2 & 3.2 & 8 & 3.9 & \\
\hline \multicolumn{8}{|l|}{ Alcoholic beverages } \\
\hline No & 100 & 71.4 & 47 & 74.6 & 147 & 72.4 & \multirow{2}{*}{0.640} \\
\hline Yes & 40 & 28.6 & 16 & 25.4 & 56 & 27.6 & \\
\hline \multicolumn{8}{|l|}{ Daily water consumption } \\
\hline No & 8 & 5.7 & 1 & 1.6 & 9 & 4.4 & \multirow{2}{*}{0.186} \\
\hline Yes & 132 & 94.3 & 62 & 98.4 & 194 & 95.6 & \\
\hline \multicolumn{8}{|l|}{ Easy access to water at school } \\
\hline No & 18 & 12.9 & 0 & 0.0 & 18 & 8.9 & \multirow{2}{*}{0.003} \\
\hline Yes & 122 & 87.1 & 63 & 100.0 & 185 & 91.1 & \\
\hline
\end{tabular}




\begin{tabular}{|c|c|c|c|c|c|c|c|}
\hline \multirow[t]{3}{*}{ General health aspects } & \multicolumn{4}{|c|}{ DSYPHONIA } & \multirow{3}{*}{ Total } & \multirow{3}{*}{$\%$} & \multirow{3}{*}{$p$-value } \\
\hline & \multicolumn{2}{|c|}{ Yes } & \multicolumn{2}{|c|}{ No } & & & \\
\hline & $n \%$ & $\%$ & $n \%$ & $\%$ & & & \\
\hline \multicolumn{8}{|l|}{ Sleeps well } \\
\hline No & 51 & 36.4 & 15 & 23.8 & 66 & 32.5 & 0.076 \\
\hline Yes & 89 & 63.6 & 48 & 76.2 & 137 & 67.5 & \\
\hline \multicolumn{8}{|l|}{ Raises voice } \\
\hline Never & 3 & 2.1 & 0 & 0.0 & 3 & 1.5 & \\
\hline Rarely & 8 & 5.7 & 7 & 11.1 & 15 & 7.4 & \\
\hline Sometimes & 39 & 27.9 & 24 & 38.1 & 63 & 31.0 & 0.173 \\
\hline Often & 58 & 41.4 & 23 & 36.5 & 81 & 39.9 & \\
\hline Always & 32 & 22.9 & 9 & 14.3 & 41 & 20.2 & \\
\hline \multicolumn{8}{|l|}{ Shouts } \\
\hline Never & 11 & 7.9 & 12 & 19.0 & 23 & 11.3 & \\
\hline Rarely & 27 & 19.3 & 17 & 27.0 & 44 & 21.7 & \\
\hline Sometimes & 74 & 52.9 & 23 & 36.5 & 97 & 47.8 & 0.071 \\
\hline Often & 22 & 15.7 & 9 & 14.3 & 31 & 15.3 & \\
\hline Always & 6 & 4.3 & 2 & 3.2 & 8 & 3.9 & \\
\hline \multicolumn{8}{|l|}{ Sings $(n=202)^{* *}$} \\
\hline Never & 12 & 8.6 & 12 & 19.0 & 24 & 11.9 & \\
\hline Rarely & 39 & 28.1 & 15 & 23.8 & 54 & 26.7 & \\
\hline Sometimes & 61 & 43.9 & 26 & 41.3 & 87 & 43.1 & 0.304 \\
\hline Often & 21 & 15.1 & 7 & 11.1 & 28 & 13.9 & \\
\hline Always & 6 & 4.3 & 3 & 4.8 & 9 & 4.5 & \\
\hline \multicolumn{8}{|l|}{ Voice loss or failure } \\
\hline Never & 7 & 5.0 & 21 & 33.3 & 28 & 13.8 & \\
\hline Rarely & 28 & 20.0 & 16 & 25.4 & 44 & 21.7 & \\
\hline Sometimes & 68 & 48.6 & 26 & 41.3 & 94 & 46.3 & 0.000 \\
\hline Often & 26 & 18.6 & 0 & 0.0 & 26 & 12.8 & \\
\hline Always & 11 & 7.9 & 0 & 0.0 & 11 & 5.4 & \\
\hline \multicolumn{8}{|l|}{ Fatigue while speaking $(n=202)^{* *}$} \\
\hline Never & 18 & 12.9 & 22 & 34.9 & 40 & 19.8 & \\
\hline Rarely & 16 & 11.5 & 11 & 17.5 & 27 & 13.4 & \\
\hline Sometimes & 58 & 41.7 & 20 & 31.7 & 78 & 38.6 & 0.001 \\
\hline Often & 31 & 22.3 & 9 & 14.3 & 40 & 19.8 & \\
\hline Always & 16 & 11.5 & 1 & 1.6 & 17 & 8.4 & \\
\hline \multicolumn{8}{|l|}{ Hoarseness } \\
\hline Never & 4 & 2.9 & 16 & 25.4 & 20 & 9.9 & \\
\hline Rarely & 21 & 15.0 & 21 & 33.3 & 42 & 20.7 & \\
\hline Sometimes & 72 & 51.4 & 22 & 34.9 & 94 & 46.3 & $<0.001$ \\
\hline Often & 33 & 23.6 & 4 & 6.3 & 37 & 18.2 & \\
\hline Always & 10 & 7.1 & 0 & 0.0 & 10 & 4.9 & \\
\hline
\end{tabular}

${ }^{* *} \mathrm{n}$ differs from 203 due responses that were crossed out, leading to the exclusion of questionnaires only for the topic in question; Chi-squared $\left(x^{2}\right)$ test with $p \leq 0.05$, with presence/absence of dysphonia.

Source: Research data

All variables were analyzed using logistic regression methods, with self-reported dysphonia by the teacher as well as dysphonia diagnosed based on clinical vocal characteristics and the presence of laryngeal lesions. Self-reported dysphonia was associated with respiratory problems (OR=3.00; $C 195 \%=1.25-7.22)$ and internal noise 
(OR=2.43; $C 195 \%=1.24-4.73)$. The use of medications $(O R=2.31 ; C I 95 \%=1.19-4.49)$ was risk factor for self-reported dysphonia. Easy access to water (OR=0.09; CI95\%=
0.01-0.79) was a protection factor for self-reported dysphonia (Table 4).

Table 4 - Multivariate and adjusted association with self-reported dysphonia among teachers from elementary school public system of Imperatriz, MA, Brazil, 2017.

\begin{tabular}{|c|c|c|c|c|c|c|c|c|}
\hline \multirow[b]{2}{*}{ Independent variable } & \multirow[b]{2}{*}{$p$-value ${ }^{a}$} & \multirow[b]{2}{*}{$O R$} & \multicolumn{2}{|c|}{$95 \% \mathrm{Cl}$ of OR } & \multirow[b]{2}{*}{$p$-value ${ }^{b}$} & \multirow[b]{2}{*}{$O R^{*}$} & \multicolumn{2}{|c|}{$95 \% \mathrm{Cl}$ of $\mathrm{OR}$} \\
\hline & & & Lower & Upper & & & Lower & Upper \\
\hline Sex (woman) & 0.439 & 1.48 & 0.55 & 3.99 & & & & \\
\hline Age group $(>30)$ & 0.543 & 0.89 & 0.62 & 1.28 & & & & \\
\hline Digestive problems (Yes) & 0.841 & 1.10 & 0.45 & 2.67 & & & & \\
\hline Respiratory problems (Yes) & 0.034 & 3.02 & 1.09 & 8.37 & 0.014 & 3.00 & 1.25 & 7.22 \\
\hline Hearing problems (Yes) & 0.764 & 0.87 & 0.35 & 2.14 & & & & \\
\hline Use of medication (Yes) & 0.070 & 2.00 & 0.94 & 4.23 & 0.014 & 2.31 & 1.19 & 4.49 \\
\hline Smokes (Yes) & 0.616 & 1.65 & 0.23 & 11.58 & & & & \\
\hline Drinks alcoholic beverages (Yes) & 0.670 & 0.83 & 0.36 & 1.94 & & & & \\
\hline Daily water intake (Yes) & 0.451 & 0.50 & 0.08 & 3.00 & & & & \\
\hline Easy access to water at school (Yes) & 0.074 & 0.12 & 0.01 & 1.23 & 0.030 & 0.09 & 0.01 & 0.79 \\
\hline Sleeps well (Yes) & 0.300 & 0.64 & 0.28 & 1.48 & & & & \\
\hline Dust (Yes) & 0.455 & 1.35 & 0.61 & 2.99 & & & & \\
\hline External noise (Yes) & 0.299 & 1.56 & 0.67 & 3.64 & & & & \\
\hline Internal noise (Yes) & 0.031 & 2.33 & 1.08 & 5.05 & 0.009 & 2.43 & 1.24 & 4.73 \\
\hline Good relationship with co-workers (Yes) & 0.124 & 8.40 & 0.56 & 126.49 & 0.145 & 5.29 & 0.56 & 49.81 \\
\hline Chalk dust (Yes) & 0.056 & 0.06 & 0.00 & 1.07 & 0.109 & 0.12 & 0.01 & 1.60 \\
\hline Ventilation (Inadequate) & 0.089 & 2.20 & 0.89 & 5.48 & 0.320 & 1.41 & 0.71 & 2.80 \\
\hline Lighting (Inadequate) & 0.261 & 1.66 & 0.69 & 4.02 & & & & \\
\hline Acoustics (Inadequate) & 0.850 & 1.08 & 0.47 & 2.51 & & & & \\
\hline Room size (Inadequate) & 0.087 & 2.08 & 0.90 & 4.82 & 0.198 & 1.63 & 0.77 & 3.44 \\
\hline Lesion & 0.374 & 1.76 & 0.51 & 6.07 & & & & \\
\hline
\end{tabular}

${ }^{*}$ Adjusted logistic regression; ${ }^{a} p$-value $<0.20 ;{ }^{b} p$-value $\leq 0.05$.

Source: Research data

Diagnosed dysphonia was associated with digestive problems $(O R=5.35 ; C 195 \%=2.39-11.96)$, external noise
$(O R=4.89 ; C 195 \%=1.87-12.78)$ and inadequate ventilation (OR=3.56; $C 195 \%=1.46-8.62)$ (Table 5).

Table 5 - Multivariate and adjusted association with diagnosed dyphonia (based on clinical vocal characteristics) among teachers from elementary school public system of Imperatriz, MA, Brazil, 2017.

\begin{tabular}{|c|c|c|c|c|c|c|c|c|}
\hline \multirow[b]{2}{*}{ Independent variable } & \multirow[b]{2}{*}{$p$-value ${ }^{a}$} & \multirow[b]{2}{*}{ OR } & \multicolumn{2}{|c|}{$95 \% \mathrm{Cl}$ of $\mathrm{OR}$} & \multirow[b]{2}{*}{$p$-value ${ }^{b}$} & \multirow[b]{2}{*}{$O R^{*}$} & \multicolumn{2}{|c|}{$95 \% \mathrm{Cl}$ of $\mathrm{OR}$} \\
\hline & & & Lower & Upper & & & Lower & Upper \\
\hline Sex (woman) & 0.774 & 1.19 & 0.37 & 3.85 & & & & \\
\hline Age group (>30) & 0.352 & 1.23 & 0.79 & 1.91 & & & & \\
\hline Digestive problems (Yes) & 0.000 & 6.43 & 2.29 & 18.06 & 0.000 & 5.35 & 2.39 & 11.96 \\
\hline Respiratory problems (Yes) & 0.486 & 1.60 & 0.43 & 5.95 & & & & \\
\hline Hearing problems (Yes) & 0.501 & 1.43 & 0.51 & 4.00 & & & & \\
\hline $\begin{array}{l}\text { Use of medication } \\
\text { (Yes) }\end{array}$ & 0.649 & 1.23 & 0.50 & 3.01 & & & & \\
\hline Smokes (Yes) & 0.854 & 1.24 & 0.13 & 12.13 & & & & \\
\hline Drinks alcoholic beverages (Yes) & 0.929 & 0.96 & 0.35 & 2.62 & & & & \\
\hline Daily water intake (Yes) & 0.184 & 0.22 & 0.02 & 2.07 & 0.750 & 0.77 & 0.154 & 3.85 \\
\hline Easy access to water at school (Yes) & 0.938 & 1.07 & 0.19 & 6.06 & & & & \\
\hline Sleeps well (Yes) & 0.886 & 1.08 & 0.39 & 2.95 & & & & \\
\hline Dust (Yes) & 0.676 & 0.82 & 0.31 & 2.12 & & & & \\
\hline External noise (Yes) & 0.017 & 3.71 & 1.27 & 10.88 & 0.001 & 4.89 & 1.87 & 12.78 \\
\hline Internal noise (Yes) & 0.347 & 1.58 & 0.61 & 4.06 & & & & \\
\hline Good relationship with co-workers (Yes) & 0.873 & 0.77 & 0.03 & 19.89 & & & & \\
\hline Chalk dust (Yes) & 0.780 & 0.67 & 0.04 & 10.97 & & & & \\
\hline Ventilation (Inadequate) & 0.004 & 6.01 & 1.79 & 20.23 & 0.005 & 3.56 & 1.46 & 8.62 \\
\hline Lighting (Inadequate) & 0.219 & 1.96 & 0.67 & 5.72 & & & & \\
\hline
\end{tabular}


Fernanda Aguiar da Cruz et al.

\begin{tabular}{|c|c|c|c|c|c|c|c|c|}
\hline \multirow[b]{2}{*}{ Independent variable } & \multirow[b]{2}{*}{$p$-value ${ }^{a}$} & \multirow[b]{2}{*}{$O R$} & \multicolumn{2}{|c|}{$95 \% \mathrm{Cl}$ of OR } & \multirow[b]{2}{*}{$p$-value ${ }^{b}$} & \multirow[b]{2}{*}{$O R^{*}$} & \multicolumn{2}{|c|}{$95 \% \mathrm{Cl}$ of $\mathrm{OR}$} \\
\hline & & & Lower & Upper & & & Lower & Upper \\
\hline Acoustics (Inadequate) & 0.063 & 2.63 & 0.95 & 7.28 & 0.078 & 2.15 & 0.91 & 5.05 \\
\hline Room size (Inadequate) & 0.029 & 3.30 & 1.13 & 9.64 & 0.429 & 0.70 & 0.29 & 1.69 \\
\hline Lesion & 0.998 & 1.00 & 0.00 & & & & & \\
\hline
\end{tabular}

${ }^{*}$ Adjusted logistic regression; ${ }^{a} p$-value $<0.20 ;{ }^{b} p$-value $\leq 0.05$.

Source: Research data

Laryngeal lesions were positively associated with the inadequate lighting $(O R=3.74 ; C I 95 \%=1.15-12.14)$, inadequate acoustics (OR=4.14; $C 195 \%=1.32-12.95)$ and inadequate room size (OR=3.76; $C 195 \%=1.29-10.91)$.
Respiratory problems (OR=0.21; CI95\% $=0.06-0.76)$, easy access to water $(O R=0.12 ; \mathrm{Cl} 195 \%=0.04-0.38)$ and use of medication (OR=0.34; $\mathrm{Cl} 95 \%=0.12-0.92)$ were protection factors against laryngeal lesions (Table 6).

Table 6 - Multivariate and adjusted association with laryngeal lesions among teachers in the municipal education system of Imperatriz, MA, Brazil, 2017.

\begin{tabular}{|c|c|c|c|c|c|c|c|c|}
\hline \multirow[b]{2}{*}{ Independent variable } & \multirow[b]{2}{*}{$p$-value $e^{a}$} & \multirow[b]{2}{*}{ OR } & \multicolumn{2}{|c|}{$95 \% \mathrm{Cl}$ of $\mathrm{OR}$} & \multirow[b]{2}{*}{$p$-value $e^{b}$} & \multirow[b]{2}{*}{$O R^{*}$} & \multicolumn{2}{|c|}{$95 \% \mathrm{Cl}$ of $\mathrm{OR}$} \\
\hline & & & Lower & Upper & & & Lower & Upper \\
\hline Sex (woman) & 0.080 & 23.17 & 0.69 & 777.17 & 0.056 & 4.15 & 0.96 & 17.84 \\
\hline Age group (>30) & 0.632 & 0.88 & 0.52 & 1.49 & & & & \\
\hline Digestive problems (Yes) & 0.152 & 3.12 & 0.66 & 14.80 & 0.193 & 2.58 & 0.62 & 10.73 \\
\hline Respiratory problems (Yes) & 0.102 & 0.30 & 0.07 & 1.27 & 0.017 & 0.21 & 0.06 & 0.76 \\
\hline Hearing problems (Yes) & 0.124 & 0.38 & 0.11 & 1.30 & 0.089 & 0.39 & 0.13 & 1.15 \\
\hline Use of medication (Yes) & 0.093 & 0.40 & 0.14 & 1.16 & 0.033 & 0.34 & 0.12 & 0.92 \\
\hline Smokes (Yes) & 0.999 & 0.00 & 0.00 & & & & & \\
\hline Drinks alcoholic beverages (Yes) & 0.814 & 0.87 & 0.27 & 2.78 & & & & \\
\hline Daily water intake (Yes) & 0.377 & 0.44 & 0.07 & 2.74 & & & & \\
\hline Easy access to water at school (Yes) & 0.052 & 0.23 & 0.05 & 1.01 & 0.000 & 0.12 & 0.04 & 0.38 \\
\hline Sleeps well (Yes) & 0.921 & 0.95 & 0.31 & 2.85 & & & & \\
\hline Dust (Yes) & 0.055 & 3.10 & 0.98 & 9.87 & 0.182 & 1.96 & 0.73 & 5.26 \\
\hline External noise (Yes) & 0.505 & 0.68 & 0.21 & 2.14 & & & & \\
\hline Internal noise (Yes) & 0.044 & 3.65 & 1.04 & 12.84 & 0.107 & 2.39 & 0.83 & 6.88 \\
\hline Good relationship with co-workers (Yes) & 0.243 & 0.15 & 0.01 & 3.70 & & & & \\
\hline Chalk dust (Yes) & 0.999 & 0.00 & 0.00 & & & & & \\
\hline Ventilation (Inadequate) & 0.798 & 1.16 & 0.37 & 3.65 & & & & \\
\hline Lighting (Inadequate) & 0.089 & 3.08 & 0.84 & 11.26 & 0.028 & 3.74 & 1.15 & 12.14 \\
\hline Acoustics (Inadequate) & 0.060 & 3.26 & 0.95 & 11.18 & 0.015 & 4.14 & 1.32 & 12.95 \\
\hline Room size (Inadequate) & 0.048 & 3.17 & 1.01 & 9.98 & 0.015 & 3.76 & 1.29 & 10.91 \\
\hline
\end{tabular}

${ }^{*}$ Adjusted logistic regression; ${ }^{a} p$-value $<0.20 ;{ }^{b} p$-value $\leq 0.05$.

Source: Research data

Among the 60 participants who underwent video-assisted laryngoscopy, $53.3 \%(n=32)$ has some type of laryngeal lesion and $43.3 \%(n=26)$ had no lesions but had other findings. Moreover, $3.3 \%(n=2)$ were unable to do the exam due to feelings of nausea. During the performance of video-laryngoscopies, the entire oral and laryngeal cavity of the teachers was evaluated, and thus other findings located outside the vocal folds were identified. However, as the objective of the study was to study changes that fit dysphonia, only laryngeal lesions were considered among the findings. Among these videolaryngoscopic findings, the laryngeal lesions found were as follows: micronodules (21.7\%), vocal nodules (8.3\%), vascular dysgenesis (8.3\%), vocal sulcus (5\%), thickening of free edge of the left vocal fold (1.7\%) and right vocal fold (3.3\%), leukoplakia (3.3\%) and aryepiglottic fold cyst (1.7\%). The prevalence of laryngeal lesions among the participants who underwent laryngoscopy was 53.3\% (data not presented in table).

\section{DISCUSSION}

The main findings of the present study were the high prevalence of dysphonia among teachers and the fact that environmental factors related to the workplace can lead to occupational health problems.

It is necessary to understand the context of worker health among teachers in order to achieve significant improvements in the working conditions of these professionals as well as in the context of Brazilian Public Health. The present findings suggest the need to investigate services offered to teachers in the public education system in different regions of Brazil and determine factors associated with vocal disorders in this occupation.

This study shows high prevalence rates of self-reported dysphonia (68.9\%), of diagnosed dysphonia (59.1\%) 
and laryngeal lesions in teachers with dysphonia (53.3\%) who work in the basic education system in a municipality in Amazonia. Multicausality was found for vocal disorders, with an increased chance of self-reported or diagnosed (based on four or more clinical vocal characteristics or video-assisted laryngoscopy) dysphonia in teachers with respiratory and digestive problems and those who teach in an inadequate work environment. However, the use of medications, respiratory problems and easy access to water had protective effects.

The prevalence of vocal disorders was higher than rates reported in developed countries such as Europe and the United States of America ${ }^{7,10,16}$, as well as other national studies $^{12,28,29}$, although literature describes extreme variability regarding disorders in this occupation ${ }^{9}$.

In the present study, vocal problems were more frequent among the female teachers, who accounted for $86.3 \%$ of the individuals with such problems. The literature ${ }^{30-33}$ also reports that women are more vulnerable to vocal problems due to anatomical-physiological aspects of the larynx specific to the female sex ${ }^{34}$, the vocal frequency similar to that found in children, double-work shifts ${ }^{35}$ and level of schooling ${ }^{5}$. In elementary education, the proportion of women largely prevails. While the male gender has a greater participation in secondary and higher education. This can be a good example that the higher the educational level, the greater the number of men involved ${ }^{36}$.

Age merits particular attention. In the present study, the large portion of the sample was between 50 and 59 years of age (40.6\%), followed by the 40-to-49-year-old age group (38.2\%), who were precisely those most affected by vocal problems (31.4 and 39.4\%, respectively). Studies on the voice report that 24 to 45 years is considered the age group with the best vocal efficiency and the chances of structural laryngeal changes that affect vocal quality increase with the advance of age ${ }^{28}$.

No statistically significant associations were found between dysphonia and sociodemographic variable (age group, sex, marital status, schooling and teaching experience), which agrees with findings described in previous studies $^{26,31}$. A high percentage of teachers had a complete university education (62.1\%). This finding may be related to education guidelines stipulated in legislation in Brazil (Law no 9.394/1996), which establishes that educators with an undergraduate or graduate degree can teach on all levels of the education system, including basic education ${ }^{32}$.

The internal noise in the work environment was pointed out by most of the dysphonic teachers (68.6\%), and our work showed that the presence of noise in the classroom had a significant association with voice problems. Previous studies report a similar finding $31,37,38$. A survey conducted in Dublin identified that $71.3 \%$ of elementary school teachers worked in classes with more than 30 students, and among this group, $69.4 \%$ had vocal disorders, also highlighted that the working conditions of these teachers need to be fully adapted to promote vocal healt ${ }^{39}$. In this sense, we suggest the use of amplifiers and microphones as important aspects of individual protective equipment ${ }^{5}$ in environments that are considered aggravating to the vocal health of teachers as a way to minimize speech effort and vocal fatigue ${ }^{40,41}$. Nebulization with $0.9 \% \mathrm{NaCl}$ (NEB) to protect the teacher's voice in the workplace ${ }^{42}$, as well as vocal warm-up/cool-down and the semi-occluded vocal tract exercise with commercial straw, are also low-cost strategies, with positive effects on the teacher's voice in the workplace ${ }^{43}$.

External noise was also positively associated with vocal symptoms, which agrees with a study conducted in 12 public schools in Colombia (OR=1.83; C195\%=1.12-2.99) ${ }^{35}$. School noise is present in different forms both inside and outside the classroom, but internal noise is more perceptible to students and teachers than external noise ${ }^{39-41}$. Brazilian Norm NBR 10152 of the Brazilian Association of Technical Standards stipulates a noise level of 35 to 45 $d B$ as acceptable for the classroom ${ }^{44}$. A study conducted in a city in the state of São Paulo ${ }^{45}$ portrays this situation well, as the mean environmental noise in the classroom ranged from 40 and $51 \mathrm{~dB}$ without the students and from 45 to $65 \mathrm{~dB}$ with the students, accompanied by an intensity of the voice of the teacher ranging from 52 to $68 \mathrm{~dB}$, reaching $7.8 \mathrm{~dB}$ above the environmental noise. In the study, $90 \%$ of the schools investigated had noise levels above those recommended by NBR 10152. Another study highlights the reference to noise as something that can be subjective and this aspect should ideally be evaluated in an objective manner, correlating it with the degree of intelligibility of the teacher's speaking ${ }^{16}$. In the present study, other environmental factors (inadequate lighting, acoustics, ventilation and classroom size) associated with vocal disorders concomitantly to exposure to noise further aggravated the working environment of teachers. These factors can also have consequences for the respiratory system, thereby exerting an impact on vocal production.

The municipality of Imperatriz is located in the state of Maranhão, which has the second worst Human Development Index in the northeastern region of the country ${ }^{25}$. The region is characterized by a wet tropical climate with high temperatures as well as environmental pollution and the burning of biomass in the second semester of the year ${ }^{31}$. Classroom infrastructure is precarious, and many urban streets are unpaved, which have impacts on the working and health conditions of the entire school community. These data are compatible to those reported in a study conducted in Nigeria ${ }^{10}$.

A significant association was found between dysphonia and digestive problems. Among the participants in the present study, $74.4 \%$ had some type of complaint related to the gastrointestinal tract. The most common symptoms were heartburn (60.1\%) and acid reflux (41.8\%). Moreover, signs of laryngopharyngeal reflux were found in $86.7 \%$ of the teachers who were submitted to video-assisted laryngoscopy, despite being an atypical form of gastroesophageal reflux disease, this fact may be related 
to the environmental exposures in the workplace, such as: "burning" and dust from the existing unpaved streets. The literature also reports high prevalence rates of acid reflux, with 70 to $90 \%$ of vocal problems in professionals who rely on their voices due to both vocal activity and living habits, such as smoking, alcohol use and a sedentary lifestyle $26,27,46$.

A significant association was also found between dysphonia and respiratory problems. Approximately, $86.2 \%$ of the teachers reported a history of respiratory conditions, such as rhinitis, sinusitis, pharyngitis, laryngitis, bronchitis/asthma and colds, the most prevalent of which were colds (66.9\%), sinusitis (45.8\%), rhinitis (36.9\%) and tonsillitis (36.9\%), which is similar to data reported in previous studies ${ }^{10,47}$. Among allergic disorders, rhinitis occurs repeatedly and its main cause is inhaled allergens, such as dust mites, mold dust and chalk dust; moreover, allergic rhinitis is an aggravating factor of dysphonia and teachers are advised to seek specific treatment to avoid a worsening of the condition 32,48 .

The use of medications in the previous two weeks was also positively associated with vocal problems. This practice was common among $46.8 \%$ of the teachers evaluated and $52.1 \%$ of those with dysphonia. No detailed investigation was made regarding what medications were used or the duration of treatment (acute or continuous). Therefore, information bias may have occurred. It should also be pointed out that the use of medication does not necessarily mean that these individuals sought a physician, as they may have been self-medicating. The question about drug treatment refers to the use of general medication. Perhaps, this association is related to the increase in the use of psychotropic drugs by teachers and the association between voice problems and mental disorders ${ }^{49}$, mainly among teachers who work in various schools ${ }^{50}$.

Another finding was that teachers with dysphonia and respiratory problems had a lower chance of exhibiting vocal lesions. It is possible that the symptoms of a laryngopathy emerge more rapidly and more intensively in the presence of an aggravating factor among individuals with a previously established laryngeal problem, making them quickly diminish the demands on their voice, which may explain the protective effect found in this study. Another question, maybe this finding in our study may be explained by the fact of use of medication also being a protective factor for vocal lesions, taking into consideration that some of these medicines have been used to treat respiratory problems of the teachers. However, this hypothesis should be investigated later.

The consumption of water during work was frequent among the teachers, with 95.6\% drinking water during their activities on daily basis and $91.1 \%$ reporting having easy access to water in the work environment. Easy access to water was also a vocal protection factor, which agrees with findings describe in previous studies ${ }^{50}$, demonstrating the importance of water to the maintenance and im- provement of vocal health. Therefore, facilitating access to water is a simple, low-cost measure that should be performed and encouraged in all vocal health policies.

The present study has limitations that should be considered. In the cross-sectional design, exposure and outcome factors are determined simultaneously, which does not enable the determination of causality between vocal disorders and associated factors. Therefore, one cannot discard the possibility of measurement and recall bias. Most teachers did not undergo video-assisted laryngoscopy and the extrapolation of the data to all teachers in basic education should be performed with caution. Moreover, the prevalence of vocal problems may have been underestimated due to the non-perception of such problems or a lack of knowledge regarding one's own voice ${ }^{34}$.

\section{CONCLUSION}

The present study reveals a high prevalence of vocal disorders among elementary school teachers of Imperatriz, state of Maranhão, located in Brazil's Legal Amazon (BLA). Dysphonia and laryngeal lesions were evaluated using three methods: self-perceptions, diagnostic criteria, and video-assisted laryngoscopy. Vocal disorders were associated with aspects related to the working environment, such as internal noise, external noise, respiratory problems, digestive problems, and the use of medications. Among the teachers with dysphonia, respiratory problems, use of medication and easy access to water during work activities were protection factors. The results of this study underscore the need for the combined effort of worker health, education and public health administrators for the establishment of public policies aimed at the promotion of occupational health that prioritize actions on different levels, such as awareness regarding the voice as an instrument of work and an important teaching tool, investment in regular vocal improvement programs for teaching staff and the drafting of strategies that can minimize factors associated with risk to the vocal health of teachers. Moreover, there is an urgent need to implement public policies that promote vocal health, to prevent morbidities, and to improve working conditions in this occupation.

\section{REFERENCES}

1. PAN, B. et al. Factors Associated with Job Satisfaction among University Teachers in Northeastern Region of China: a cross-sectional study. Int. J. Environ. Res. Public. Health, Basel, v.12, n.10, p.12761-12775, 2015.

2. SILVA, L. G.; SILVA, M. C. Condições de trabalho e saúde de professores pré-escolares da rede pública de ensino de Pelotas, RS, Brasil. Ciênc. Saúde Colet., Rio de Janeiro, v.18, n.11, p.3137-3146, 2013.

3. SANTOS, L. R. et al. Adherence of the dysphonic teachers in speech therapy. CoDAS, São Paulo, v.25, n.2, p.134-139, 2013.

4. ALRAHIM, A. A.; ALANAZI, R. A.; AL-BAR, M. H. Hoarseness among school teachers: A cross-sectional study from Dammam. J. Family Community Med., [s.I], v.25, n.3, p.205-210, 2018. 
5. MOY, F. M. et al. Determinants and effects of voice disorders among secondary school teachers in peninsular Malaysia using a validated Malay version of VHI-10. PLoS ONE, San Francisco, v.10, n.11, p.e0141963, 2015.

6. KIM, K. H. et al. Prevalence of and sociodemographic factors related to voice disorders in South Korea. J. Voice, New York, v.30, n.2, p.24612467, 2016.

7. GIANNINI, S. P. P.; LATORRE, M. R. D. O.; FERREIRA, L.P. Distúrbio de voz e estresse no trabalho docente: um estudo caso-controle. Cad. Saúde Pública, Rio de Janeiro, v. 28, n.11, p.2115-2124, 2012.

8. MANOEL, A. L. et al. Alterações laríngeas em professores do ensino fundamental: prevalência, fatores de risco e achados da videolaringoscopia. Rev. AMRIGS, Porto Alegre, v. 60, n.2, p.115-120, 2016.

9. FILLIS, M. M. A. et al. Frequência de problemas vocais autorreferidos e fatores ocupacionais associados em professores da educação básica de Londrina, Paraná, Brasil. Cad. Saúde Pública, Rio de Janeiro, v.32, n.1, p.e00026015, 2016.

10. AKINBODE, R. et al. Voice disorders in Nigerian primary school teachers. Occup. Med., Chicago, v. 64. n.5, p.382-386, 2014.

11. QUINTAIROS, S. Incidências de nódulos vocais em professores de pré-escola e o seu tratamento. Rev. CEFAC, São Paulo, v.2, p.16-22, 2000.

12. BEHLAU, M. et al. Epidemiology of voice disorders in teachers and nonteachers in Brazil: prevalence and adverse effects. J. Voice, New York, v.26, n.5, p.665.e9-18, 2012.

13. GIANNINI, S. P. P.; LATORRE, M. R. D. O. FERREIRA, L. P. Distúrbios de voz relacionado ao trabalho docente: um estudo caso-controle. CoDAS, São Paulo, v. 25, n.6, p. 566-576, 2013.

14. PIZOLATO, R. A. et al. Evaluation of risk factors for voice disorders in teachers and vocal acoustic analysis as an instrument of epidemiological assessment. Rev. CEFAC, São Paulo, v.15, n.4, p. 957-966, 2013.

15. LOPEZ, M. M. et al. Bem-estar e fatores associados em professores do ensino fundamental no sul do Brasil. Rev. CEFAC, São Paulo, v.19, n.6, p.812-820, 2017.

16. CUTIVA, L. C. C.; VOGEL, I.; BURDORF, A. Voice disorders in teachers and their associations with work-related factors: a systematic review. J. Commun. Disord., Amsterdam, v.46, n.2, p.143-155, 2013.

17. FERRACCIU, C. C. S. et al. Estratégias de enfrentamento e perfil de participação e atividades vocais em professoras da rede pública de ensino com e sem distúrbios de voz. Rev. CEFAC., São Paulo v.17, n.4, p.1184-1194, 2015.

18. MEDEIROS, A. M.; VIEIRA, M. T. Distúrbio de voz como doença relacionada ao trabalho no Brasil: reconhecimento e desafios. Cad. Saúde Pública, Rio de Janeiro, v. 35, n.10, p.e00174219, 2019.

19. MASSON, M. L.V.; FABBRON, E. M. G.; LOIOLA-BARREIRO, C. M. Aquecimento e desaquecimento vocal em professores: estudo quase-experimental controlado. CoDAS, São Paulo, v.31, n.4, p.e20180143, 2019.

20. ASSOCIAÇÃO BRASILEIRA DE OTORRINOLARINGOLOGIA E CIRURGIA CÉRVICO-FACIAL (ABORL-CCF). et al. Consenso Nacional sobre Voz Profissional. 2004. Disponível em: https://www.ablv.com.br/ wp-content/uploads/2020/09/consenso_voz_profissional.pdf. Acesso em: 03 dez 2020.

21. MASSON, M. L. V. et al. Disturbio de voz: reconhecimento revogado junto com a nova lista de doenças relacionadas ao trabalho. Rev. Bras. Saúde Ocup., São Paulo, v.45, n.E32, 2020.

22. SERVILHA, E. A. M.; CORREIA, J. M. Correlation between environment, Work organization conditions, voice symptoms self-reported by university professors and speech-language pathology assessment. Distúr. Comum., São Paulo, v.26, n.3, p.452-462, 2014.

23. BOLBOL, S. A. et al. Risk factors of voice disorders and impact of vocal hygiene awareness program among teachers in public schools in Egypt. J. Voice, New York, v.31, n.2, p.e9-251.e316, 2017.

24. MARTINS, G. et al. Voice Disorders in Teachers. A Review. J. Voice, New York, v. 28, n.6, p.9, 2014.

25. INSTITUTO BRASILEIRO DE GEOGRAFIA E ESTATÍSTICA (IBGE). Censo 2019. Cidades e Estados. Maranhão, 2019. Disponível em: < https:// www.ibge.gov.br/cidades-e-estados/ma.html>. Acesso em: 07 out. 2020.

26. ALVES, L. P.; ARAÚJO, L. T. R.; XAVIER NETO, J. A. Prevalência de queixas vocais e estudo de fatores associados e uma amostra de professores de ensino fundamental em Maceió, Alagoas, Brasil. Rev. Bras. Saúde Ocup., São Paulo, v.35, n.121, p.168-75, 2010.

27. VALENTE, A. M. S. L.; BOTELHO, C.; SILVA, A. M. C. Distúrbio de voz e fatores associados em professores da rede pública. Rev. Bras. Saúde Ocup., São Paulo, v. 40, n. 132, p. 183-195, 2015.

28. LOPES, M. C. L. A. et al. Fatores associados à saúde vocal e a qualidade de vida em professores. Rev. CEFAC, São Paulo, v.20, n.4, p.515-531, 2018.

29. ASSUNÇÃO, A. A. et al. Occupational and individual risk factors for dysphonia in teachers. Occup. Med., Chicago, v.62, n.7, p.553-559, 2012.

30. SOUZA, C. L. et al. Fatores associados a patologia de pregas vocais em professores. Rev. Saúde Pública, São Paulo, v.45, n.5, p.914-21, 2011.

31. VALENTE, A. M. S. L.; BOTELHO, C.; SILVA, A. M. C. Distúrbios de voz e fatores associados em professores da rede pública. Rev. Bras. Saúde Ocup., São Paulo, v.40, n.132, p.183-195, 2015.

32. SILVA, G. J. et al. Sintomas vocais e causas autorreferidas em professores. Rev. CEFAC, São Paulo, v.18, n.1, p.158-1, 2016.

33. MARTINS, R. H. et al. Voice disorders in teachers. A review. J. Voice., New York, v.28, n.6, p.716-24, 2014.

34. ALMEIDA, L. M. S.; DUMITH, S. C. Associação entre problemas vocais e tempo de trabalho em servidores públicos de uma Universidade Federal do Sul do Brasil. Cad. Saúde Colet., Rio de Janeiro, v.26, n.3, p.249-254, 2018

35. CUTIVA, L. C. C; BURDORF, A. Effects of noise and acoustics in schools on vocal health in teachers. Noice Health, [s.I], v.17, n.74, p.17-22, 2015.

36. ARAUJO, T. M.; PINHO, P. S.; MASSON, M. L. V. Trabalho e saúde de professoras e professores no Brasil: reflexões sobre trajetórias das investigações, avanços e desafios. Cad. Saúde Pública, Rio de Janeiro, v. 35, p.e00087318, 2019.

37. BUTLER, J. E.; HAMMOND, T. H.; GRAY, S. D. Genderrelated differences of hialuronic acid distribution in the human vocal fold. Laryngoscope, St. Louis, v.111, n.5, p.907-911, 2011.

38. SERVILHA, E. A. M.; RUELA, I. S. Riscos ocupacionais à saúde e voz de professores: especificidades das unidades de rede municipal de ensino. Rev. CEFAC, São Paulo, v.12, n.1, p.109-114, 2010.

39. MUNIER, C.; FARRELL, R. Working conditions and workplace barriers to vocal health in primary school teachers. J. Voice, New York, v.30, n.1, p.e127-e131, 2015.

40. BOVO, R. et al. Voice amplification for primary school teachers with voice disorders: a randomized clinical trial. Int. J. Occup. Med. Environ. Health, Lodz, v.26, n.3, p.363-72, 2013.

41. TEIXEIRA, L. C.; BEHLAU, M. Comparison Between Vocal Function 
Exercises and Voice Amplification. J. Voice, New York, v.29, n.6, p.71826, 2015.

42. MASSON, M. L. V.; ARAÚJO, T. M. Protective Strategies Against Dysphonia in Teachers: Preliminary Results Comparing Voice Amplification and $0.9 \% \mathrm{NaCl}$ Nebulization. J. Voice, New York, v.32, n.2, p.257. e1-257.e10, 2018.

43. SOUZA, R. C.; MASSON, M. L. V.; ARAUJO, T. M. Efeitos do exercício do trato vocal semiocluído em canudo comercial na voz do professor. Rev. CEFAC, v. 19, n. 3, p. 360-370, 2017.

44. ASSOCIAÇÃO BRASILEIRA DE NORMAS TÉCNICAS (ABNT). NBR 10152: Níveis de Pressão Sonora em Ambientes Internos a Edificações. Brasil, 2017.

45. GUIDINI, R. F. et al. Correlações entre ruído ambiental em sala de aula e voz do professor. Rev. Soc. Bras. Fonoaudiol., v.17, n.4, p.398404, 2012.
46. CHEN, S. H. et al. Risk factors and effects of voice problems for teachers. J. Voice, v.2, p.183-192, 2010.

47. SIQUEIRA, M. A. et al. Hidratação vocal em profissionais e futuros profissionais da voz. Rev CEFAC, São Paulo, v. 18, n.4, p.908-914, 2016.

48. ROY, N. et al. Voice disorders in teachers and the general population: effects on work performance, attendance, and future career choices. J. Speech Lang. Hear. Res., Rockville, v.47, n.3, p.542-551, 2004.

49. TOSTES, M. V. et al. Sofrimento mental de professores do ensino público. Saúde em Debate, Londrina, v.42, n.116, p.87-99, 2018.

50. RODRÍGUEZ-LOUREIRO, L. et al. Joint effect of paid working hours and multiple job holding on work absence due to health problems among basic education teachers in Brazil: the Educatel Study. Cad. Saúde Pública, Rio de Janeiro, v.35, p.e00081118, 2019.

Submetido em: $27 / 11 / 2019$

Aceito em: 03/02/2021 